Tropical Journal of Pharmaceutical Research June 2012; 11 (3): 379-386

(C) Pharmacotherapy Group, Faculty of Pharmacy, University of Benin

Benin City, 300001 Nigeria.

All rights reserved.

Available online at http://www.tjpr.org

Research Article

http://dx.doi.org/10.4314/tjpr.v11i3.6

\title{
In-vitro Release Study of Carvedilol Phosphate Matrix Tablets Prepared with Hydroxypropyl Methylcellulose
}

\author{
Shimul Halder ${ }^{1}$, Maruf Hasan ${ }^{1,}$ Biplab Kumar Das ${ }^{2 *}$, Abul Kalam Lutful \\ $\mathrm{Kabir}^{1}$ and Abu Shara Shamsur Rouf ${ }^{1 *}$ \\ ${ }^{1}$ Department of Pharmaceutical Technology, Faculty of Pharmacy, University of Dhaka, Dhaka-1000, ${ }^{2}$ Department of \\ Pharmacy, North South University, Dhaka-1229, ${ }^{3}$ Department of Pharmacy, Jagannath University, Dhaka-1000, \\ Bangladesh.
}

\begin{abstract}
Purpose: To evaluate the effect of two molecular weight grades of hydroxypropyl methylcellulose on the release characteristics of carvedilol phosphate matrix tablets.

Methods: Matrix tablets containing carvedilol phosphate were prepared from 27 formulations in three batch series coded $A, B$ and $C$, each containing 9 formulations. Each batch incorporated different ratios of two molecular weight grades of hydroxypropyl methylcellulose (Methoce ${ }^{\circledR}$ K4M CR and K15M CR) used as release retarding agents. Microcrystalline cellulose (Avicel PH 101), starch (Sta-Rx 1500) and lactose monohydrate were used as diluents in the formulations while the effect of sodium lauryl sulphate (wetting agent) was studied for some of the formulations. The tablets were characterized for carvedilol phosphate release in both simulated gastric and intestinal fluids. The data were subjected to different models in order to determine their release kinetics and mechanisms.

Results: All the batches released more than $50 \%$ of their carvedilol content in $12 h$ when Methoce ${ }^{\circledR}$ K4M CR and K15M CR constituted $18 \%$ and $15 \%$ of the matrix, respectively. Avice ${ }^{\beta}$ PH 101 decreased while Starch 1500 and lactose monohydrate increased drug release. Drug release mechanism was predominantly diffusion.

Conclusion: By using varying combinations of two molecular weight grades of hydroxypropyl methylcellulose as the matrix, controlled or sustained release carvedilol tablets of varying release characteristics can be prepared.
\end{abstract}

Keywords: Carvedilol phosphate, Matrix tablet, Hydroxypropyl methylcellulose, Release kinetics 


\section{INTRODUCTION}

Some of the goals in designing controlled release drug delivery systems (CRDDS) are to reduce the frequency of drug administration, provide uniform drug delivery and facilitate drug targeting [1]. The use of controlled release (CR) formulations offers many potential advantages, such as sustained blood levels, attenuation of adverse effects and improved patient compliance. It is important especially in the case of antihypertensive agents to maintain constant blood levels, as otherwise dose dumping may cause hypotension.

Carvedilol is chemically (+)-1-(Carbazol-4yloxy)-3- $\{[2-(0-m e t h o x y$ phenoxy) ethyl] amino\}-propan-2-ol and is an antihypertensive drug with multiple mechanisms of action. It acts as a non-selective $\beta$ and $\alpha-1$ adrenergic receptor blocker and it also has vasodilating property that is attributed mainly to its $\alpha-1$ receptor antagonist activity $[2,3]$. Its conventional tablet dosage form is used to treat mild-to-moderate hypertension and angina pectoris [4]. Carvedilol base is practically insoluble in water $(0.583 \mathrm{mg} / \mathrm{L})$ and thus poorly absorbed from the gastrointestinal tract. It exhibits poor absolute bioavailability of $25-35 \%$ [5]. The half-life of the drug is $6-8 \mathrm{~h} \mathrm{[6]}$. Its very poor aqueous solubility indicates that its absorption is dissolution rate-limited which results in irregular and delayed absorption. Therefore conventional tablets are required to be administered 3 - 4 times a day. A suitable sustained release dosage form of carvedilol should provide prolonged action and better compliance by the patient. Recently, a phosphate salt of carvedilol was developed with improved aqueous solubility and chemical stability by protonation of the secondary amine as a salt [7].

Methocel derivatives have been widely used in the design of complex controlled release systems because of their low toxicity, $\mathrm{pH}$ independent swelling and drug embedding ability [8]. These polymers are hydrophilic in nature and can retain active ingredients within its scaffold. Its retention ability, however, depends on the concentration or ratio of polymers used [9]. HPMC 4 and HPMC 15 are two typically used methocel polymers for the formulation of hydrophilic matrix systems, providing a robust mechanism for the slow release of drugs from oral solid dosage forms. They are suitable for preparing formulations with soluble or insoluble drugs and at high or low dosage levels. Hydration of polymers results in the formation of a gel layer that controls the release rate of drug from the core of matrix tablets [10].

The aim of this work was to prepare matrix tablets containing carvedilol phosphate as a model drug, as well as either of two molecular weight grades (4 or 15 cps viscosity grade) hydroxypropyl methylcellulose (HPMC) as matrix formers to control drug release. The mechanism of drug release was also determined using various kinetic models

\section{EXPERIMENTAL}

Carvedilol phosphate (Aravinda Pharmaceuticals Limited, India) was a generous gift from Beximco Pharmaceuticals Limited, Bangladesh. Methocel ${ }^{\circledR}$ K4M CR and K15M CR, Avicel PH101, starch (Sta-Rx 1500, Colorcon,India), talc, and magnesium stearate were obtained from Dow Chemical's Asia Pvt, Limited, India. All other chemicals were of analytical grade and were used as received.

\section{Preparation of carvedilol phosphate matrix tablets}

The carvedilol phosphate matrix tablets were prepared according to the formulations shown in Table 1. Drug, polymers and other excipients were weighed separately for 50 tablets per formulation for 27 proposed formulations in three batch series (each batch consists of 9 formulations) coded as A (A1-A9), B (B1-B9) and $C$ (C1-C9). The amount of carvedilol 
Halder et al

Table 1: Composition of carvedilol phosphate matrix tablets

\begin{tabular}{|c|c|c|c|c|c|c|}
\hline \multirow[b]{2}{*}{ Batch code } & \multicolumn{6}{|c|}{ Amount of Ingredients (mg) } \\
\hline & $\begin{array}{c}\text { HPMC } 4 \\
\text { cps grade* }\end{array}$ & $\begin{array}{c}\text { HPMC } 15 \\
\text { cps grade* }\end{array}$ & $\begin{array}{c}\text { Avicel (PH } \\
\text { 101) }\end{array}$ & $\begin{array}{c}\text { Starch } \\
\text { (Sta-Rx } \\
1500) \\
\end{array}$ & $\begin{array}{c}\text { Lactose } \\
\text { mono } \\
\text { hydrate }\end{array}$ & $\begin{array}{c}\text { Sodium } \\
\text { lauryl } \\
\text { sulfate }\end{array}$ \\
\hline A1 & 37.5 & 37.5 & 131.3 & 0 & 0 & 0 \\
\hline A2 & 30 & 37.5 & 138.8 & 0 & 0 & 0 \\
\hline A3 & 45 & 37.5 & 122.5 & 0 & 0 & 1.25 \\
\hline A4 & 37.5 & 30 & 138.75 & 0 & 0 & 0 \\
\hline A5 & 37.5 & 45 & 123.8 & 0 & 0 & 0 \\
\hline A6 & 45 & 30 & 130 & 0 & 0 & 1.25 \\
\hline A7 & 30 & 45 & 131.3 & 0 & 0 & 0 \\
\hline A8 & 30 & 30 & 146.3 & 0 & 0 & 0 \\
\hline A9 & 45 & 45 & 115 & 0 & 0 & 1.25 \\
\hline B1 & 37.5 & 37.5 & 0 & 131.3 & 0 & 0 \\
\hline B2 & 30 & 37.5 & 0 & 138.8 & 0 & 0 \\
\hline B3 & 45 & 37.5 & 0 & 122.5 & 0 & 1.25 \\
\hline B4 & 37.5 & 30 & 0 & 138.75 & 0 & 0 \\
\hline B5 & 37.5 & 45 & 0 & 123.8 & 0 & 0 \\
\hline B6 & 45 & 30 & 0 & 130 & 0 & 1.25 \\
\hline B7 & 30 & 45 & 0 & 131.3 & 0 & 0 \\
\hline B8 & 30 & 30 & 0 & 146.3 & 0 & 0 \\
\hline B9 & 45 & 45 & 0 & 115 & 0 & 1.25 \\
\hline C1 & 37.5 & 37.5 & 0 & 0 & 131.3 & 0 \\
\hline $\mathrm{C} 2$ & 30 & 37.5 & 0 & 0 & 138.8 & 0 \\
\hline C3 & 45 & 37.5 & 0 & 0 & 122.5 & 1.25 \\
\hline C4 & 37.5 & 30 & 0 & 0 & 138.75 & 0 \\
\hline C5 & 37.5 & 45 & 0 & 0 & 123.8 & 0 \\
\hline C6 & 45 & 30 & 0 & 0 & 130 & 1.25 \\
\hline $\mathrm{C} 7$ & 30 & 45 & 0 & 0 & 131.3 & 0 \\
\hline C8 & 30 & 30 & 0 & 0 & 146.3 & 0 \\
\hline C9 & 45 & 45 & 0 & 0 & 115 & 1.25 \\
\hline
\end{tabular}

* Percent ratios of HPMC 4 and HPMC 15 used were: i. 15\%: 15\%, ii. 12\%: 15\%, iii. 18\%: 15\%, iv. 15\%: 12\% v. $15 \%: 18 \%$ vi. $18 \%: 12 \%$, vii. $12 \%$ : $18 \%$ viii. $12 \%: 12 \%$ ix. $18 \%$ : $18 \%$ of the total weight of the formulation

phosphate, talc and Mg-stearate used in each formulation was $40 \mathrm{mg}, 1.25 \mathrm{mg}$ and $2.5 \mathrm{mg}$, respectively, while the weight of each tablet was $250 \mathrm{mg}$. The required amounts of drugs and excipients were mixed together for 15 min in a mixer; talc was added and mixed for another $1 \mathrm{~min}$. The blended mass was transferred to the hopper of a laboratory hydraulic press fitted with a $10 \mathrm{~mm}$ flat-faced punch and die sets adjusted to a compressing weight of $250 \mathrm{mg}$. The tablets were compressed using a compression force and compression time of 5 tons and $20 \mathrm{~s}$, respectively. Prior to compression, the die and punch surfaces were sufficiently lubricated with magnesium stearate.

\section{In vitro release studies}

In vitro drug release studies of the matrix tablets were carried out using a six-station USP XXII type II dissolution test apparatus (Eurolab, Germany) at $37 \pm 0.5{ }^{\circ} \mathrm{C}$ and 100 rpm speed in $900 \mathrm{ml}$ of $0.1 \mathrm{M}$ hydrochloric acid (gastric simulated fluid, $\mathrm{pH} 1.3$ ) as a dissolution medium for the first $2 \mathrm{~h}$ and in intestinal simulated fluid $(900 \mathrm{ml}, \mathrm{pH} \mathrm{6.8)}$ for the next $10 \mathrm{~h}$.

Simulated gastric fluid (SGF) was prepared by diluting $11.4 \mathrm{ml}$ of hydrochloric acid (32\%, $\mathrm{w} / \mathrm{v}$ ) with sufficient distilled water to make up to $1000 \mathrm{ml}$. One tablet from each formulation was placed in a basket and the equipment 
was operated. Samples $(10 \mathrm{ml})$ were withdrawn hourly over a period of $2 \mathrm{~h}$, and the samples filtered The volume of dissolution medium was replenished with 10 $\mathrm{ml}$ of fresh dissolution medium. The absorbance of the samples was measured with a single-beam UV-spectrophotometer (Shimadzu UV-1601, Japan) at $241 \mathrm{~nm}$ and the amount of drug released calculated. The release studies were conducted in triplicate, and mean values of drug released were plotted against time.

After the $2 \mathrm{~h}$ test in SGF, $20 \mathrm{ml}$ of $\mathrm{NaOH}(25$ $\%$ ) was added to the SGF fluid and the $\mathrm{pH}$ adjusted to $6.8 \pm 0.05$ by the addition of 1.2 $\mathrm{ml}$ ortho-phosphoric acid $(1 \mathrm{~N})$ to the remaining SGF fluid The operation was continued for $10 \mathrm{~h}$, and samples were withdrawn, filtered and analysed as described for the test in SGF.

\section{Kinetic analysis of release data and mechanism of drug release}

In order to evaluate the kinetics and the mechanism of drug release from the formulations, the data obtained from the in vitro drug release studies were analyzed by zero-order [11] (cumulative percent of drug released vs time, Eq 1) and the Higuchi model [12] (cumulative percent of drug released vs square root of time) shown in Eq 2

$M_{t}=M_{0}+k_{0} t$

$M_{t}=M_{0}+k_{H} t^{1 / 2}$

where $M_{t}$ is the cumulative amount of drug released at any time, $t$, and $M_{0}$ the dose of the drug incorporated in the delivery system, and $k_{0}$ and $k_{H}$ are the rate constants for zeroorder and Higuchi models, respectively.

The dissolution data were also fitted to the well-known exponential equation of Peppas et al [13] ( $\mathrm{Eq} \mathrm{3)}$ which is often used to describe drug release behavior from polymeric systems.

$M_{t} / M \square=k t^{n}$
Where, $M_{t} / M$ is the fraction of drug released at time, $t, k$ is the kinetic constant, and $n$ is the diffusional exponent for drug release. The diffusional exponent, $n$, is dependent on the geometry of the device as well as the physical mechanism for release. A value of $n$ $=0.45$ indicates Fickian (case I) release; > 0.45 but $<0.89$ for non-Fickian (anomalous) release; and $>0.89$ indicates super case II type of release. Case II generally refers to the erosion of the polymeric chain and anomalous transport (Non-Fickian) refers to a combination of both diffusion and erosioncontrolled drug release [14].

\section{Statistical analysis}

Data from the experiments were statistically evaluated by 2-tailed regression analysis using the Statistical Package for Social Science (SPSS) software for Windows (version 17, SPSS Inc., Chicago, Illinois, USA), and the multiple coefficient values $\left(R^{2}\right.$ value) for non-linear drug release versus time. Square root or log time relationship was calculated and used as the parameter for determining statistical significance. Statistical analysis of the results was performed by using one-way analysis of variance (ANOVA) and the limit of significance was set at $p<$ 0.05 .

\section{RESULTS}

\section{Drug release from matrix tablets}

The cumulative percent of carvedilol phosphate release from the matrix tablets of batch series ' $A$ ', ' $B$ ' and ' $C$ ' are shown in Figures 1, 2 and 3 . The release data for formulations, A1, B1 and C1 showed 65.3, 65.9 and $73.4 \%$ drug release, respectively, in $12 \mathrm{~h}$ where the percent ratio of HPMC 4 and HPMC 15 in the formulations were 1:1 ratio. Besides, the tablets of batch series "B" and " $C$ " showed increased drug release compared to the tablets of batch series "A". Starch 1500 and lactose monohydrate were used as the diluents for batch " $B$ " and " $C$ ", respectively. The release rates attained a 
value of more than $50 \%$ in $12 \mathrm{~h}$ when either HPMC 4 and HPMC 15 constituted at least $15 \%$ of the matrix tablet. The tablets of formulations A3, A6 and A9 containing sodium lauryl sulfate showed increased dissolution rate which was mostly noticeable in the formulations where both polymers constituted $18 \%$ of the matrix tablet. However, the dissolution rate was highest in formulations B3, B6, B9 and C3, C6, C9 compared to $A 3, A 6$ and $A 9$. The release data obtained were fitted into various release kinetic models and the outcome is listed in Table 2.
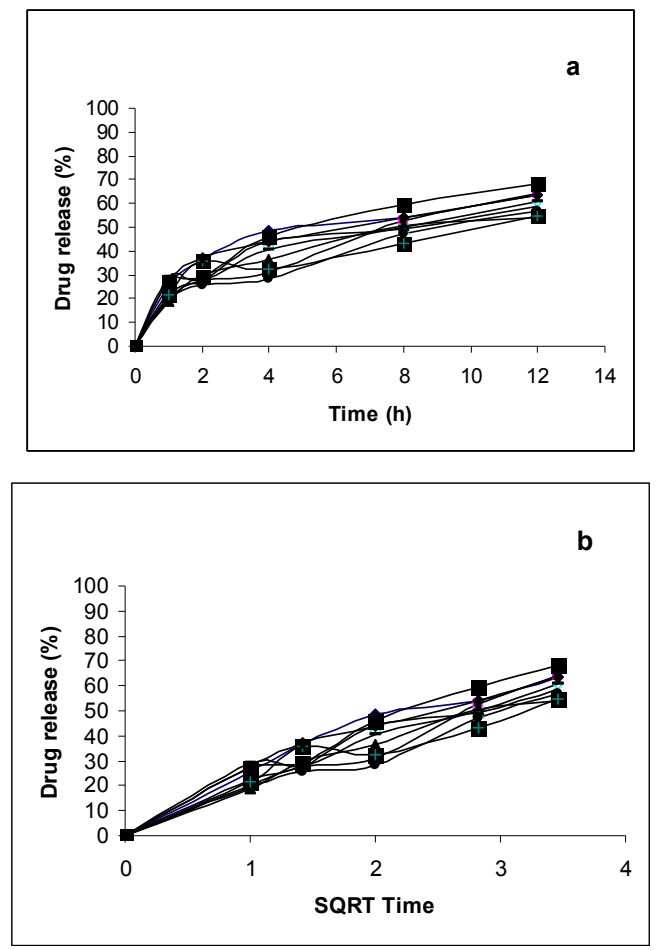

Figure 1: Comparative release profile of carvedilol phosphate from matrix tablets based on (a) zero order and (b) Higuchi model. Key: batches A1 ( $\downarrow)$,

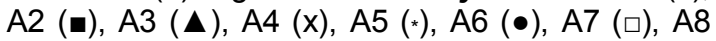
$(\cdot), A 9(-)$

\section{DISCUSSION}

Carvedilol phosphate is an important drug in the treatment of hypertension and stable angina pectoris. Besides its antioxidant and antiproliferative effects, it is reported to enhance one's ability to combat the deleterious effects of sympathetic nervous system activation in heart failure $[15,16]$. In view of its superiority in the treatment of hypertension, the preparation of a suitable sustained release dosage form might increase the efficacy of treatment and patient compliance by producing desirable blood concentrations and by minimizing the incidence of adverse effects.
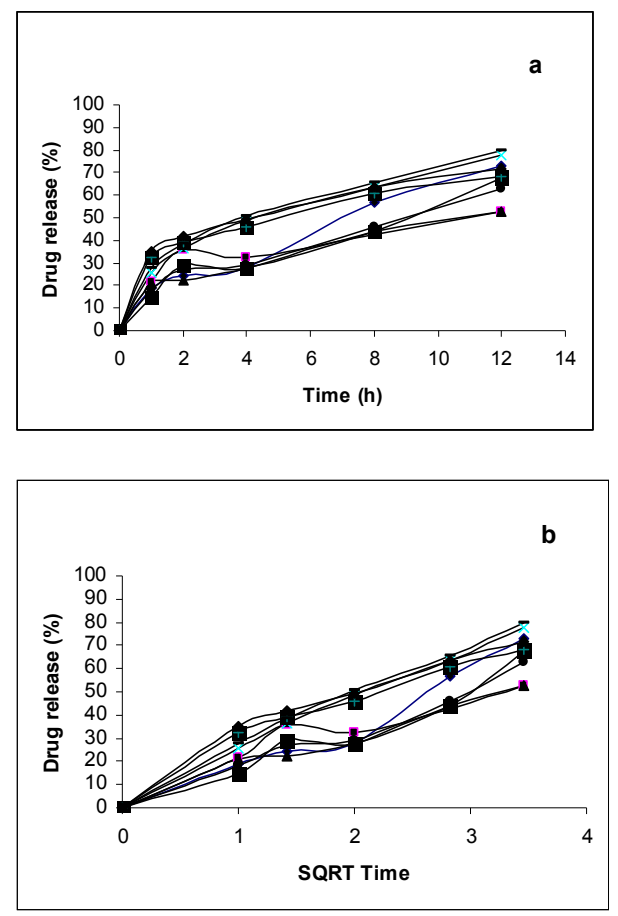

Figure 2: Comparative release profile of carvedilol phosphate from matrix tablets based on (a) zero order and (b) Higuchi model. Key: batches B1 ( $\downarrow)$, B2 (๘), B3 $(\mathbf{\Delta})$, B4 (x), B5 $(*)$, B6 $(\bullet)$, B7 (), B8 $(\cdot)$, B9 $(-)$.

As there are no sustained release tablets of carvedilol phosphate available in the Bangladesh market, theoretical sustained release needed for once-daily (12-24 h) administration was calculated based on its pharmacokinetics as suggested by Wagner et al [17] and the release profiles of the formulated tablets were compared with the theoretical sustained release needed to select the optimized formulation. A once-daily 

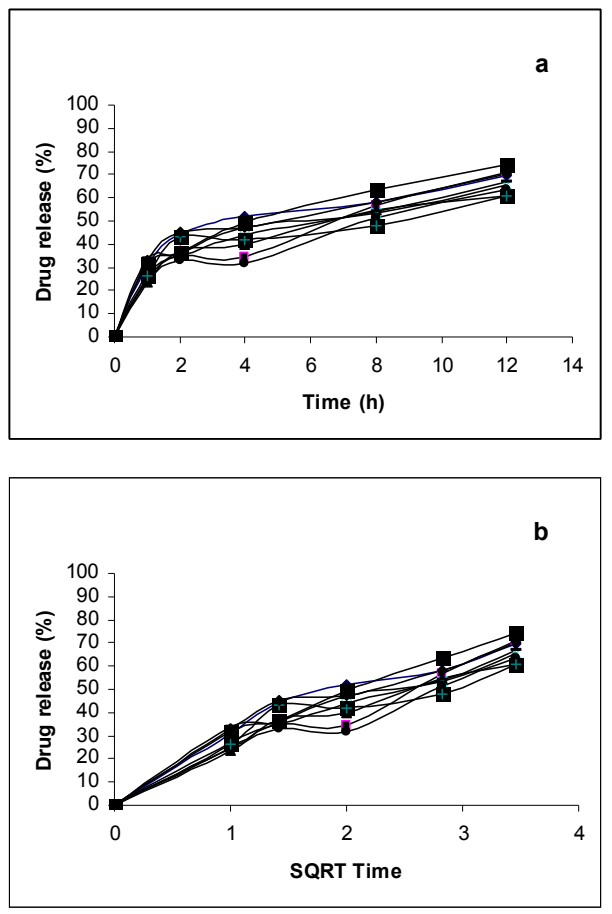

Figure 3: Comparative release profile of carvedilol phosphate from matrix tablets based on (a) zero order and (b) Higuchi model. Key: batches C1 ( $\bullet$ ),

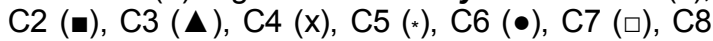
(•), C9 (-)

controlled release product of carvedilol phosphate should contain a total dose of 40 $\mathrm{mg}$ (initial dose - $20.5 \mathrm{mg}$; maintenance dose - $19.5 \mathrm{mg}$ ) and the drug should be released at a rate $\left(k_{0}\right)$ of $2.475 \mathrm{mg} / \mathrm{h}$. Based on these doses and release rate $\left(k_{0}\right)$, an oral CR tablet of carvedilol phosphate should provide a release of $28.8 \%$ in $1 \mathrm{~h}, 31.9 \%$ in $2 \mathrm{~h}, 38.1$ $\%$ in $4 \mathrm{~h}, 50.4 \%$ in $8 \mathrm{~h}, 62.8 \%$ in $12 \mathrm{~h}$, and $100 \%$ in $24 \mathrm{~h}[18,19]$. The similarity factor (based on fit factor test) with theoretical profile was calculated and found to be 64.4 , indicating good similarity. The rate of release of the formulations mostly complied with the theoretical sustained release formulation. The release rate of carvedilol phosphate from the tablets of batch series ' $B$ ' and ' $C$ ' increased as Starch 1500 and lactose monohydrate may act as disintegrants. Sodium lauryl sulfate was used as a surfactant to increase the dissolution rate of the matrix tablets; it indeed improved drug release rate in formulations C3, C6 and C9 compared to the formulations of ' $A$ ' and ' $B$ ' series and this may be due to the greater hydrophilic property of lactose monohydrate compared to microcrystalline cellulose and Starch 1500. The presence of sodium lauryl sulfate didn't seem to be essential for an ideal formulation because formulations $\mathrm{A} 5, \mathrm{~B} 5$ and $\mathrm{C} 5$ showed maximum drug release of $70.1,70.7$ and $78.2 \%$, respectively, after $12 \mathrm{~h}$ compared to other formulations. This indicates that for effective controlled drug release, HPMC must constitute a minimum of $18 \%$ of the tablet formulation.

From the correlation coefficient values, Higuchi model was the best-fitting model for carvedilol phosphate release since higher

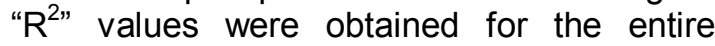
release process (see table 2 ), which indicates a diffusion-controlled release mechanism. The average $R^{2}$ values in Higuchi kinetics for the tablets of batches A, B and C were 0.969 , 0.971 and 0.957 , respectively, which were statistically significant at $p \leq 0.01$ levels. On the other hand, the average $R^{2}$ values (for zero-order kinetics) for the tablets of batches $\mathrm{A}, \mathrm{B}$ and $\mathrm{C}$ were $0.831,0.835$ and 0.801 , respectively, which were statistically significant at $p \leq 0.05$ levels. Diffusion was related to transport of the drug from the dosage matrix into the dissolution fluid, depending on the concentration [20]. As drug concentration gradient rose, the drug diffusion pathway increased. This could explain why the drug diffused at a comparatively slower rate as the distance for diffusion increased, which is related to the square-root component of the Higuchi model[12]. In this work, the in vitro drug profiles of all the formulations could be best expressed by Higuchi model as the plots showed high linearity $\left(R^{2}=0.9793\right.$ to 0.9922).

To confirm it is diffusion-control mechanism, the data were fitted to Korsmeyer's model [20]. Almost all the formulations showed good 
Halder et al

Table 2: Drug release kinetics for batch series "A", "B" and "C" matrix tablets

\begin{tabular}{clccccc}
\hline $\begin{array}{c}\text { Batch code } \\
\text { of } \\
\text { formulation }\end{array}$ & \multicolumn{3}{c}{ Zero-order } & \multicolumn{2}{c}{ Higuchi } & \multicolumn{2}{c}{ Korsmeyer-Peppas } \\
\cline { 2 - 7 } $\mathbf{R}^{2}$ & & $\left.\mathbf{k}_{\mathbf{0}} \mathbf{( h}^{-1}\right)$ & $\mathbf{R}^{2}$ & $\left.\mathbf{k}_{\mathbf{H}} \mathbf{h}^{-1 / 2}\right)$ & $\mathbf{R}^{2}$ & $\begin{array}{c}\text { Diffusional } \\
\text { exponent (n) }\end{array}$ \\
\hline A1 & 0.761 & 4.423 & 0.951 & 18.24 & 0.967 & 0.357 \\
A2 & 0.926 & 4.947 & 0.988 & 18.85 & 0.969 & 0.476 \\
A3 & 0.832 & 4.152 & 0.981 & 16.63 & 0.980 & 0.437 \\
A4 & 0.818 & 4.329 & 0.971 & 17.40 & 0.968 & 0.415 \\
A5 & 0.854 & 4.923 & 0.983 & 20.21 & 0.957 & 0.418 \\
A6 & 0.909 & 5.104 & 0.987 & 16.59 & 0.962 & 0.441 \\
A7 & 0.777 & 3.715 & 0.934 & 15.03 & 0.887 & 0.335 \\
A8 & 0.783 & 4.410 & 0.962 & 18.03 & 0.995 & 0.330 \\
A9 & 0.822 & 4.285 & 0.964 & 17.13 & 0.913 & 0.340 \\
B1 & 0.766 & 4.495 & 0.954 & 18.50 & 0.970 & 0.363 \\
B2 & 0.927 & 5.019 & 0.988 & 19.12 & 0.971 & 0.482 \\
B3 & 0.835 & 4.224 & 0.981 & 16.89 & 0.980 & 0.443 \\
B4 & 0.822 & 4.402 & 0.972 & 17.66 & 0.969 & 0.421 \\
B5 & 0.856 & 5.170 & 0.987 & 20.47 & 0.957 & 0.424 \\
B6 & 0.911 & 4.389 & 0.987 & 16.85 & 0.964 & 0.448 \\
B7 & 0.784 & 3.787 & 0.940 & 15.30 & 0.896 & 0.342 \\
B8 & 0.788 & 4.482 & 0.965 & 18.30 & 0.996 & 0.336 \\
B9 & 0.826 & 4.357 & 0.967 & 17.39 & 0.915 & 0.346 \\
C1 & 0.740 & 4.824 & 0.939 & 20.05 & 0.963 & 0.324 \\
C2 & 0.893 & 5.318 & 0.979 & 20.66 & 0.944 & 0.421 \\
C3 & 0.804 & 4.553 & 0.968 & 18.43 & 0.964 & 0.385 \\
C4 & 0.799 & 4.731 & 0.967 & 19.21 & 0.984 & 0.368 \\
C5 & 0.837 & 5.506 & 0.983 & 22.01 & 0.986 & 0.376 \\
C6 & 0.872 & 4.718 & 0.974 & 18.40 & 0.934 & 0.387 \\
C7 & 0.713 & 4.087 & 0.907 & 17.01 & 0.880 & 0.303 \\
C8 & 0.755 & 4.812 & 0.943 & 19.84 & 0.974 & 0.302 \\
C9 & 0.798 & 4.686 & 0.957 & 18.93 & 0.952 & 0.306 \\
\hline
\end{tabular}

$k_{0}=$ zero-order rate constant; $k_{H}=$ Higuchi rate constant; $R^{2}=$ Correlation coefficient

linearity $\left(R^{2}=0.887\right.$ to 0.996$)$, with diffusional exponent $(n)$ values ranging from 0.302 to 0.482 , indicating that diffusion was the dominant mechanism of drug release and that it was essentially quasi-Fickian diffusion (case I transport). The diffusional exponent of batches $A 2(n=0.476)$ and B2 $(n=0.482)$ indicates non-Fickian release, i.e., drug release is by coupling of Fickian diffusion and polymer matrix relaxation - the so-called anomalous diffusion; this also indicates that drug release is controlled by more than one process. Similar results were observed by Basak et al [21] with matrix tablets of ambroxol hydrochloride containing HPMC; they considered the $\mathrm{n}$ value of less than 0.5 to be indicative of quasi-Fickian diffusion mechanism.

\section{CONCLUSION}

HPMC 4 and HPMC 15 in varying proportions controlled carvedilol phosphate release effectively for $12 \mathrm{~h}$. thus, the formulations should be suitable as a twice daily sustained release carvedilol phosphate tablet. In most cases, the release kinetics of carvedilol phosphate from the matrix tablets followed Higuchi kinetics. Furthermore, the rate of drug release is affected by the use of different diluents in the formulations.

\section{ACKNOWLEDGEMENT}

The authors wish to thank Beximco Pharmaceuticals Limited, Dhaka, Bangladesh, for providing carvedilol 
phosphate and also Center for Excellence, University of Dhaka, for technical support.

\section{REFERENCES}

1. Merkus FWHM. Rate- Controlled Drug Administration and Action, Boca Raton, FL, USA, CRC Press. 1986, pp 15-47.

2. Nicholas AJ, Sulpizio AC, Ashton DJ, Hieble JP, Ruffolo RR Jr. In vitro pharmacologic profile of the novel beta-adrenoreceptor antagonist and vasodialator, carvedilol. Pharmacology 1989; 39: 327-336.

3. Nicholas AJ, Gellai M, Ruffolo RR Jr: Studies on the mechanism of arterial vasodialation produced by the novel anti hypertensive agent, carvedilol. Fundamental Clinical Pharmacology 1991; 5: 25-38.

4. Tenero DM, Henderson LS, Charlotte A, Baidoo MM. Pharmacokinetic properties of a new controlled release carvedilol. The Amer $J$ Cardiol 2006; 98: 5L-16L.

5. Kalimuthu S, Yadav A V. Formulation and evaluation of carvedilol loaded Eudragit E 100 nanoparticles. International J Pharm Tech Res 2009; 1(2): 179-183.

6. McTavish D, Campoli-Richards D, Sorkin EM. Carvedilol: A review of its pharmacodynamic and pharmacokinetic properties and therapeutic efficacy. Drugs 1993; 45: 232-258.

7. Chakarborty S, Shukla D, Jain A, Mishra B, Singh S. Assesment of solubilization characteristics of different surfactant for carvedilol phosphate as a function of $\mathrm{pH}$. J Col and Interface Sci 2009; 335: 242-249.

8. Reza, SM, Quadir, MA, Haider, SS. Comparative evaluation of plastic, hydrophobic and hydrophilic polymers as matrices for controlled-release drug delivery. J Pharm Sci, 2003; 6(2): 282-291.

9. Aulton Me, Razzak A, Hogan JE. The mechanical properties of Hydroxypropyl methyl cellulose films derived from aqueous systems. Part I. Aulton ME. Pharmaceutics: the

science of dosage form design, 1981, pp 191211.
10. Peppas, NA, Sahlin, JJ. Hydrogels as mucoadhesive and bioadhesive materials: a review. Biomaterials, 1996, 17(16): 1553-1561.

11. Harland R, Dubernet C, Benoit JP, Peppas NA. A Model of dissolution-controlled, diffusional drug release from non-swellable polymeric microspheres. J Control Rel, 1988; 7: 207215.

12. Higuchi T. Mechanism of sustained action medication: Theoretical analysis of rate of release of solid drugs dispersed in solid matrices. J Pharm Sci, 1963; 52: 1145-1149.

13. Ritger PL, Peppas NA. A simple equation for description of solute release II. Fickian and anomalous release from swellable devices. $J$ Control Rel, 1987; 5: 37-42.

14. Donglu S. Biomedical Devices and Their Applications. Beijing, China, Springer, 2004, pp 1-31.

15. Feuerstein GZ, Rufflo RR Jr. Carvedilol, a novel multiple action antihypertensive agent with antioxidant activity and the potential for myocardial and vascular protection. European Heart J, 1995; 16:38-42.

16. Sung $C P$, Arleth AJ, Ohlstein EH: Carvedilol inhibits vascular smooth muscle cell proliferation. J Cardio Pharmacol 1993; 21:221-227.

17. Wagner JG, Nelson E. Percent absorbed time plots derived from blood level and/or urinary excretion data. J Pharm Sci, 1963; 52: 610611.

18. McTavish D, Campoli-Richards D, Sorkin EM. Carvedilol: A review of its pharmacodynamic and pharmacokinetic properties and therapeutic efficacy. Drugs 1993; 45: 232-258.

19. Ruffolo RR Jr, Gellai M, Hieble JP, Willette RN, Nichols AJ. The pharmacology of carvedilol Eur J Clin Pharmacol 1990;38: S82-88.

20. Korsmeyer RW, Gurny R, Doelker E, Buri P, Peppas NA. Mechanism of solute release from porous hydrophilic polymers. Int $\mathrm{J}$ Pharma 1983; 15: 25-35.

21. Basak SC, Jayakumar Reddy BM, Lucas Mani KP. Formulation and release behavior of sustained release ambroxol hydrochloride HPMC matrix tablet. Indian J Pharm Sci 2006; 68: $594-598$ 